\title{
Relaxation of $\mathrm{ArH}^{+}$by collision with He: Isotopic effects ${ }^{\star}$
}

\author{
Ricardo M. García-Vázquez ${ }^{1}$, Maykel Márquez-Mijares ${ }^{1}$, Jesús Rubayo-Soneira ${ }^{1}$, and Otoniel Denis-Alpizar ${ }^{2}$ \\ 1 Instituto Superior de Tecnologías y Ciencias Aplicadas (InSTEC), Universidad de La Habana, Ave. Salvador Allende No. 1110 , \\ Quinta de los Molinos, La Habana 10400, Cuba \\ e-mail: jrs@instec.cu \\ 2 Instituto de Ciencias Químicas Aplicadas, Facultad de Ingeniería, Universidad Autónoma de Chile, El Llano Subercaseaux 2801, \\ San Miguel, Santiago, Chile \\ e-mail: otoniel.denis@uautonoma.cl
}

Received 16 April 2019 / Accepted 9 September 2019

\begin{abstract}
Context. The study of noble gas compounds has gained renewed interest thanks to the recent detection of $\mathrm{ArH}^{+}$in the interstellar medium (ISM). The analysis of physical-chemical conditions in the regions of the ISM where $\mathrm{ArH}^{+}$is observed requires accurate collisional data of $\mathrm{ArH}^{+}$with $\mathrm{He}, \mathrm{H}_{2}$, electrons, and $\mathrm{H}$.

Aims. The main goals of this work are to compute the first three-dimensional potential energy surface (PES) to study the interaction of $\mathrm{ArH}^{+}$with $\mathrm{He}$, analyze the influence of the isotopic effects in the rate coefficients, and evaluate the rovibrational relaxation rates. Methods. Two ab initio grids of energy were computed at the coupled cluster with single, double, and perturbative triple excitations $(\mathrm{CCSD}(\mathrm{T}))$ level of theory using the augmented correlation consistent polarized quadruple, and quintuple zeta basis sets (aug-ccpVQZ, and aug-cc-pV5Z) and a grid at the complete basis set limit was determined. The analytical representation of the PES was performed using the reproducing kernel Hilbert space (RKHS). The dynamics of the system was studied using the close coupling method.

Results. The differences in the rate coefficients for the isotopes ${ }^{36} \mathrm{ArH}^{+},{ }^{38} \mathrm{ArH}^{+}$, and ${ }^{40} \mathrm{ArH}^{+}$in collision with He are negligible. However, the rotational rates for the collision of $\mathrm{ArD}^{+}$with $\mathrm{He}$ cannot be estimated from those for $\mathrm{ArH}^{+}+\mathrm{He}$. Comparison with previous rates for the ${ }^{36} \mathrm{ArH}^{+}+\mathrm{He}$ collision showed discrepancies for $|\Delta j|>2$, and in the case of high initial rotational states of ${ }^{36} \mathrm{ArH}^{+}$differences were found even for $|\Delta j|=1$. The rates for transitions between different vibrational states were also examined. Finally, new sets of rotational rates for ${ }^{36} \mathrm{ArH}^{+}+\mathrm{He}$ and ${ }^{36} \mathrm{ArD}^{+}+\mathrm{He}$ are reported.
\end{abstract}

Key words. astrochemistry - molecular data - molecular processes - scattering - ISM: molecules

\section{Introduction}

Argonium $\left(\mathrm{ArH}^{+}\right)$, the first noble molecule detected in the space, was observed through its $j=1 \rightarrow 0$ and $j=2 \rightarrow 1$ emission in the Crab nebula (Barlow et al. 2013). After this detection, $\mathrm{ArH}^{+}$ has been observed in several sources of the diffuse interstellar medium (ISM) (Schilke et al. 2014) and in extragalactic regions (Müller et al. 2015). Now it is known that noble gas compounds can be formed in nature, which increases the interest in these systems (Novak \& Fortenberry 2016).

The $\mathrm{ArH}^{+}$molecules can be produced in the ISM from the reactions below (Barlow et al. 2013; Bop et al. 2017):

$\mathrm{Ar}^{+}+\mathrm{H}_{2} \rightarrow \mathrm{ArH}^{+}+\mathrm{H}$

$\mathrm{Ar}+\mathrm{H}_{2}^{+} \rightarrow \mathrm{ArH}^{+}+\mathrm{H}$

$\mathrm{Ar}+\mathrm{H}_{3}^{+} \rightarrow \mathrm{ArH}^{+}+\mathrm{H}_{2}$,

but they are principally formed by reaction (1) after the ionization of Ar by cosmic rays or secondary photons produced by cosmic rays (Schilke et al. 2014; Priestley et al. 2017; Dagdigian 2018). The formation of the $\mathrm{ArD}^{+}$is also possible from the deuterated species of reactions (1) and (3) (Anicich 1993).

The determination of the physicochemical conditions in the ISM can be estimated from local thermal equilibrium(LTE). In the LTE model, the population of the rotational states of the observed

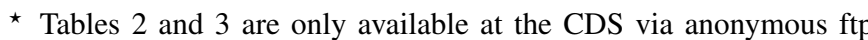
to cdsarc.u-strasbg. fr $(130.79 .128 .5)$ or via http://cdsarc. u-strasbg.fr/viz-bin/cat/J/A+A/631/A86
}

molecule is described from a Boltzmann distribution. However, in typical molecular clouds, the densities are low and collisions rare; therefore, LTE does not apply and it is necessary to carry out non-LTE analysis. The non-LTE models only require knowledge of the Einstein coefficients and state-to-state inelastic rate coefficients with the most common colliders in the ISM (e.g., $\mathrm{He}, \mathrm{H}_{2}$, electrons, and $\mathrm{H}$ ). Usually, the Einstein coefficients are known, but this is not the case for the collisional rates. The emission lines observed for $\mathrm{ArH}^{+}$are the result of collisions with electrons or with $\mathrm{H}_{2}$ molecules (Barlow et al. 2013). In addition, according to Loh et al. (2012) electrons are ten times less abundant than hydrogen in any form in the molecular region of the Crab nebula. It was also observed in this reference that the ratio of intensity $\mathrm{I}\left(\mathrm{H}_{2} 2.12 \mu \mathrm{m}\right) / \mathrm{I}\left(\mathrm{H}_{\mathrm{I}} \mathrm{Br} \gamma\right)$ is also 10 . These facts make the study of the collision of $\mathrm{ArH}^{+}$with $\mathrm{H}_{2}$ molecules important. There is particular interest in the collision with $\mathrm{He}$ because the collisional data for the interaction with $\mathrm{H}_{2}$ can be evaluated from those with He using a mass scaling factor (Schöier et al. 2005). Even if in the case of collisions with ions this approximation is questionable due to the differences in the long-range of the PES for collisions with $\mathrm{He}$ and $\mathrm{H}_{2}$, the propensity rules are expected to be correctly described. Furthermore, collision with $\mathrm{H}_{2}$ requires increasing the dimensionality of the systems and considering the formation and destruction of chemical bonds.

In reactions like Eqs. (1) and (2) the produced diatom can be formed in an excited vibrational state (Denis-Alpizar et al. 2017). The vibrational excitation of $\mathrm{ArH}^{+}$by electron-impact 
was investigated (Abdoulanziz et al. 2018); however, the only theoretical study that reports the rate coefficients for the collision of $\mathrm{ArH}^{+}$with $\mathrm{He}$ (Bop et al. 2017) was done using a two-dimensional potential energy surface (PES) at the explicitly correlated coupled cluster with single, double, and perturbative triple excitations (CCSD(T)-F12) level of theory where the diatomic molecule was considered as a rigid rotor. To our knowledge, a full three-dimensional PES for the $\mathrm{ArH}^{+}+\mathrm{He}$ complex does not exist; therefore, the vibrational relaxation for this collision has not been considered so far.

Furthermore, two different isotopes, ${ }^{36} \mathrm{ArH}^{+}$and ${ }^{38} \mathrm{ArH}^{+}$, have been detected in the ISM (Schilke et al. 2014). Even if the effects on the collisional rate coefficients for the change of isotopes of Ar are not expected to be high as a consequence of the small change in the reduced mass and shift of the center of mass in the diatom $\mathrm{ArH}^{+}$, they deserve to be evaluated. The case of the change of H by D could be different. Significant discrepancies have been found in the rates for the collisions with $\mathrm{He}$ in others studies, for example, for $\mathrm{HCO}^{+} / \mathrm{DCO}^{+}$(Buffa 2012; Buffa et al. 2009), NH/ND (Dumouchel et al. 2012), HCN/DCN (Denis-Alpizar et al. 2015), and $\mathrm{CH}^{+} / \mathrm{CD}^{+}$(Werfelli et al. 2017).

The main goals of the present work are to develop the first three-dimensional PES for the interaction of $\mathrm{ArH}^{+}$with $\mathrm{He}$, analyze the influence of the isotopic effects in the rates for this collision, and evaluate the rovibrational rate coefficients. The methods used in this work are described in Sect. 2, while the results are presented and discussed in Sect. 3. Finally, the main conclusions are summarized in Sect. 4.

\section{Ab initio calculations and scattering}

\subsection{Ab initio energies}

The ab initio calculations for the $\mathrm{ArH}^{+}+\mathrm{He}$ system were performed at the $\operatorname{CCSD}(\mathrm{T})$ level of theory using the augmented correlation consistent polarized quadruple, and quintuple zeta basis sets (aug-cc-pVQZ, and aug-cc-pV5Z) of Dunning (Dunning 1989) as implemented in the Molpro package (Werner et al. 2012). The energies at the complete basis set limit (CBS) were determined by $E_{\mathrm{CBS}}=\frac{E_{X} X^{3}-E_{Y} Y^{3}}{X^{3}-Y^{3}}$, where $X=5$ and $Y=4$ and $E_{X}$ and $E_{Y}$ are the energies computed at the respective cardinality of the basis set (Halkier et al. 1999). This level of theory is comparable to the CCSD(T)-F12/aug-cc-pVTZ method employed by Bop et al. (2017), but without the inaccuracy of the F12 method in the long range. The Jacobi coordinates $(r, R, \theta)$ were used, where the linear $\mathrm{ArH}^{+}-\mathrm{He}$ corresponds to $\theta=0^{\circ}$. The basis set superposition error was corrected at all geometries for both grids using the counterpoise method of Boys \& Bernardi (1970),

$V^{\mathrm{int}}(r, R, \theta)=E_{[\mathrm{ArH}-\mathrm{He}]^{+}}(r, R, \theta)-E_{\mathrm{ArH}^{+}}(r, R, \theta)-E_{\mathrm{He}}(r, R, \theta)$,

where $V^{\text {int }}(r, R, \theta)$ is the interaction energy, $E_{[\mathrm{ArH}-\mathrm{He}]^{+}}(r, R, \theta)$, $E_{\mathrm{ArH}^{+}}(r, R, \theta)$, and $E_{\mathrm{He}}(r, R, \theta)$ are the energies of $[\mathrm{ArH}-\mathrm{He}]^{+}$, $\mathrm{ArH}^{+}$, and He, respectively, computed in the full basis set of the $[\mathrm{ArH}-\mathrm{He}]^{+}$complex for every grid point. Each grid includes 11 angular points at the 11 point Gauss-Legendre quadrature angles, except for $90^{\circ}$, which was replaced by $89^{\circ}$. For the $R$ and $r$ coordinates, 38 and 6 values in the intervals [1.4,26.0] $\AA$ and $[1.12,1.47] \AA$, respectively, were considered.

\subsection{Analytical representation of the PES}

The interaction PES was represented by using a reproducing kernel Hilbert space (RKHS) (Ho \& Rabitz 1996),
$V(\boldsymbol{x})=\sum_{k=1}^{M} \alpha_{k} Q\left(\boldsymbol{x}_{k}, \boldsymbol{x}\right)$,

where $M$ is the number of ab initio points, $\boldsymbol{x}_{k}$ corresponds to a grid point $\boldsymbol{x}_{k}=\left(r_{k}, R_{k}, z_{k}\right)$ with $z=\frac{(1-\cos \theta)}{2}$, and $\boldsymbol{x}$ represents the coordinates where the PES is evaluated $\boldsymbol{x}=(r, R, z)$. The term $Q\left(\boldsymbol{x}_{k}, \boldsymbol{x}\right)$ is obtained by multiplying three one-dimensional kernels,

$Q\left(\boldsymbol{x}_{k}, \boldsymbol{x}\right)=q^{2,3}\left(R_{k}, R\right) q^{2,3}\left(r_{k}, r\right) q^{2}\left(z_{k}, z\right)$,

where $q^{2,3}$ and $q^{2}$ are the radial and angular kernels as defined by Ho \& Rabitz (1996). The coefficients $\alpha_{k}$ are determined by solving the linear system $\boldsymbol{Q}_{k l} \boldsymbol{\alpha}_{l}=\boldsymbol{V}\left(\boldsymbol{x}_{k}\right)$, where $k$ and $l$ indicate the different coordinates of the grid, $Q_{k l}$ is $Q\left(\boldsymbol{x}_{k}, \boldsymbol{x}_{l}\right)$, and $\boldsymbol{\alpha}_{l}$ is the vector with the coefficients $\alpha_{k}$. The use of the $q^{2,3}$ kernel ensures the $R^{-4}$ behavior for the extrapolated long range of the surface (Soldán \& Hutson 2000).

Furthermore, a one-dimensional diatomic PES was developed for $\mathrm{ArH}^{+}$. In this case, a set of $29 \mathrm{ab}$ initio energies from $0.88 \AA$ up to $7.00 \AA$ were computed. These energies were also fitted using a one-dimensional kernel $q^{2,3}\left(r_{k}, r\right)$. The minimum of this $V_{\mathrm{ArH}^{+}}(r)$ curve, found at $r=1.28 \AA$ and the computed dissociation energy $4.05 \mathrm{eV}$, are in excellent agreement with the experimental values $r=1.29 \AA$ (Linstrom \& Mallard 2001) and $4.1 \pm 0.1 \mathrm{eV}$ (Wyatt et al. 1975), respectively.

\subsection{Dynamics}

The dynamics of the collision was performed using the Newmat code (Stoecklin et al. 2002), which solves the rovibrational inelastic close-coupling equations in the space-fixed frame. This code was recently used to study the Ar-CO collisional system, and an excellent agreement between experiments and theoretical data was found (Mertens et al. 2017). The vibration of $\mathrm{ArH}^{+}$was treated by first solving the time-independent diatomic Shrödinger's equation using an ab initio diatomic potential and a finite basis representation of imaginary exponential, as described by Colbert \& Miller (1992). The diatomic rovibrational wave functions are then evaluated along a Gauss-Hermite grid and used to calculate the vibrational part of the intermolecular potential matrix elements (Denis-Alpizar et al. 2018).

For determining the state-to-state cross sections in the $\left[10^{-2}, 3000\right] \mathrm{cm}^{-1}$ collisional energy interval, 16 rotational levels in each of the first two vibrational states of $\mathrm{ArH}^{+}$were included in the basis. The log-derivative propagator (Manolopoulos 1988) was used to solve the close-coupling equations (Arthurs \& Dalgarno 1960) and the minimum value of the largest propagation distance was set to $50 \mathrm{a}_{0}(26.5 \AA)$. At each collisional energy, the convergence was checked for the quenching cross section as a function of the total angular momentum and maximum intermolecular distance. The state-to-state rate coefficients were then computed using the Maxwell-Boltzmann distribution for averaging the corresponding cross sections at a given temperature $T$.

\section{Results and discussion}

The grid of energies at the CCSD(T)/CBS limit was used to represent the surface (see Eq. (5)). The contour plot for the equilibrium distance of $\mathrm{ArH}^{+}\left(r_{\mathrm{e}}=1.292 \AA\right)$ is shown in Fig. 1A. Two minimums were found for the system in this configuration. The global minimum, namely $-679.1 \mathrm{~cm}^{-1}$, is located at $R=2.78 \AA$ 

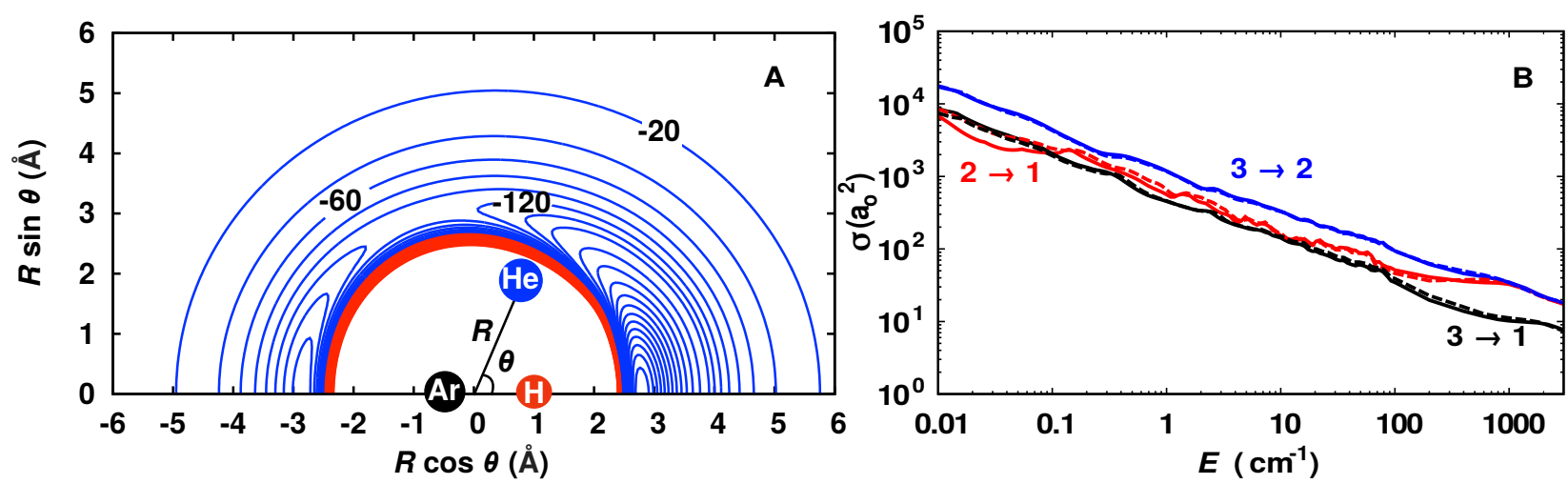

Fig. 1. Panel A: contour plot of the PES at $r_{\mathrm{e}}=1.292 \AA$. The red lines correspond to positive energies. The blue lines are represented in steps of $40 \mathrm{~cm}^{-1}$ up to $-160 \mathrm{~cm}^{-1}$ and in the step of $20 \mathrm{~cm}^{-1}$ between $-140 \mathrm{~cm}^{-1}$ and $0 \mathrm{~cm}^{-1}$. Panel $B$ : rotational de-excitation cross sections of ${ }^{36} \mathrm{ArH} \mathrm{H}^{+}+\mathrm{He}$ as functions of the collision energy including two vibrational states in the calculations (solid lines) and considering the ${ }^{36} \mathrm{ArH}^{+}$as a rigid rotor (dashed lines). Rotational transitions are labeled as $j \rightarrow j^{\prime}$.
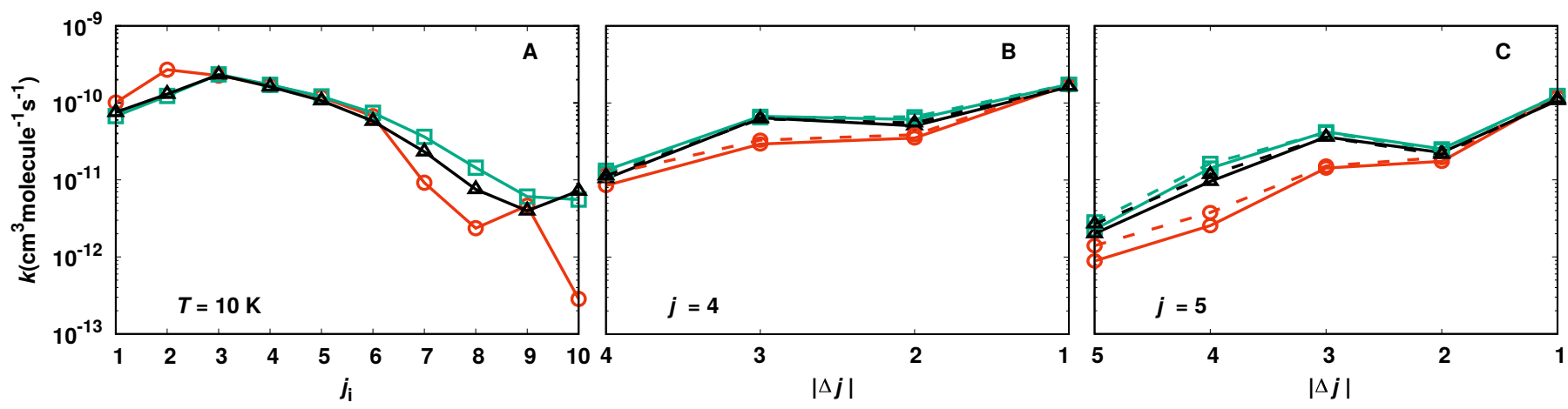

Fig. 2. Panel A: rotational de-excitation rate coefficients for the $|\Delta j|=1$ transition in the collision of ${ }^{36} \mathrm{ArH}^{+}$with He from the initial rotational state $j$, including two vibrational states in the calculations (squares) and considering the ${ }^{36} \mathrm{ArH}^{+}$as a rigid rotor (triangles) at $10 \mathrm{~K}$. The rates reported by Bop et al. (2017) are also included (circles). Panels B and $C$ : rotational de-excitation rate coefficients from the initial state $j=4$ (panel $B$ ) and $j=5$ (panel $C$ ) at $T=10 \mathrm{~K}$ (solid lines) and $T=100 \mathrm{~K}$ (dashed lines), including two vibrational states in the calculations (squares) and considering the ${ }^{36} \mathrm{ArH}^{+}$as a rigid rotor (triangles). The rates reported by Bop et al. (2017) are also included (circles).
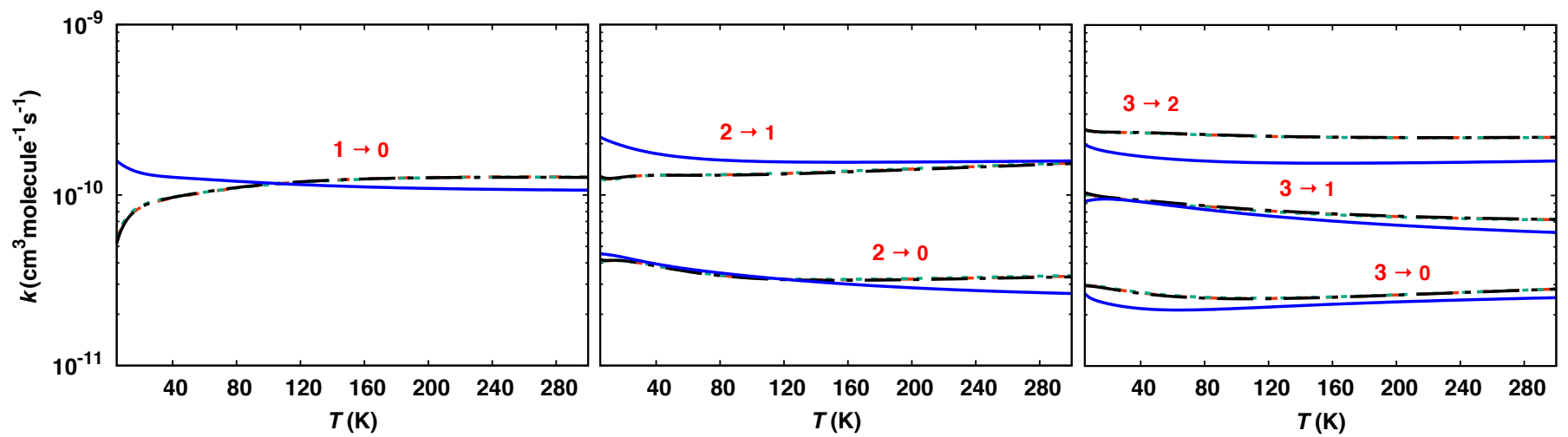

Fig. 3. Rotational rate coefficients for different isotopologs of $\mathrm{ArH}^{+}$in collision with $\mathrm{He}$ in the ground vibrational state of diatom. The solid lines represent the rates for ${ }^{36} \mathrm{ArD}^{+}+\mathrm{He}$ (blue), the dashed lines (red) indicate the rates for ${ }^{38} \mathrm{ArH}^{+}+\mathrm{He}$, and the dotted lines indicate the rates for ${ }^{40} \mathrm{ArH}^{+}+\mathrm{He}$, while dash-dotted lines (black) show the rates for ${ }^{36} \mathrm{ArH}^{+}+\mathrm{He}$. The rotational transitions are labeled as $j \rightarrow j^{\prime}$.

and $\theta=0^{\circ}$. The global minimum of the PES computed by Bop et al. (2017) is $2 \%$ deeper than that determined with our RKHS surface. The ab initio calculations of Bop et al. (2017) were performed using bond functions. In the case of calculations at the $\operatorname{CCSD}(\mathrm{T})-\mathrm{F} 12 \mathrm{a} /$ aug-cc-pVTZ level without bond functions, the difference with the minimum energy of our PES is reduced to $1.3 \%$. It is worth noticing that Burcl et al. (1995) showed that the bond functions distort the electrostatic correlation in neural systems; in the case of ionic compounds the electrostatic inter- action is larger than the dispersion energy and the effects could be larger. The secondary minimum, namely $-176.59 \mathrm{~cm}^{-1}$, is located at $R=2.83 \AA$ and $\theta=180^{\circ}$. In this case, the difference between both surfaces is lower than $0.14 \%$.

The first use of the new surface was computing the collisional rate coefficients in the ground vibrational state of ${ }^{36} \mathrm{ArH}^{+}$ for the 12 lowest rotational levels of this molecule. Two sets of calculations were performed, on one hand, considering the ${ }^{36} \mathrm{ArH}^{+}$ion as a rigid rotor and, on the other hand, including 
Table 1. Rotational and rovibrational rate coefficients $\left(\mathrm{cm}^{3} \mathrm{~mol}^{-1} \mathrm{~s}^{-1}\right)$ of ${ }^{36} \mathrm{ArH}^{+}$and ${ }^{36} \mathrm{ArD}^{+}$in collision with He at several temperatures.

\begin{tabular}{|c|c|c|c|c|c|c|c|}
\hline \multirow[b]{2}{*}{$\underline{j}$} & \multirow[b]{2}{*}{$j^{\prime}$} & \multicolumn{3}{|c|}{$\mathrm{ArH}^{+}+\mathrm{He}$} & \multicolumn{3}{|c|}{$\mathrm{ArD}^{+}+\mathrm{He}$} \\
\hline & & $k_{j, v=1 \rightarrow j^{\prime}, v^{\prime}=0}$ & $k_{j, v=0 \rightarrow j^{\prime}, v^{\prime}=0}$ & $P_{10}$ & $k_{j, v=1 \rightarrow j^{\prime}, v^{\prime}=0}$ & $k_{j, v=0 \rightarrow j^{\prime}, v^{\prime}=0}$ & $P_{10}$ \\
\hline \multicolumn{8}{|c|}{$T=100 \mathrm{~K}$} \\
\hline 1 & 0 & $2.4 \times 10^{-15}$ & $1.2 \times 10^{-10}$ & $2.1 \times 10^{-5}$ & $4.2 \times 10^{-15}$ & $1.2 \times 10^{-10}$ & $3.5 \times 10^{-5}$ \\
\hline 2 & 0 & $5.5 \times 10^{-16}$ & $3.3 \times 10^{-11}$ & $1.7 \times 10^{-5}$ & $1.9 \times 10^{-15}$ & $3.3 \times 10^{-11}$ & $5.7 \times 10^{-5}$ \\
\hline 2 & 1 & $1.5 \times 10^{-15}$ & $1.3 \times 10^{-10}$ & $1.1 \times 10^{-5}$ & $5.1 \times 10^{-15}$ & $1.6 \times 10^{-10}$ & $3.3 \times 10^{-5}$ \\
\hline 3 & 0 & $1.6 \times 10^{-16}$ & $2.5 \times 10^{-11}$ & $6.3 \times 10^{-6}$ & $9.1 \times 10^{-16}$ & $2.2 \times 10^{-11}$ & $4.2 \times 10^{-5}$ \\
\hline 3 & 1 & $4.0 \times 10^{-16}$ & $8.4 \times 10^{-11}$ & $4.8 \times 10^{-6}$ & $2.4 \times 10^{-15}$ & $7.9 \times 10^{-11}$ & $3.1 \times 10^{-5}$ \\
\hline 3 & 2 & $5.5 \times 10^{-16}$ & $2.3 \times 10^{-10}$ & $2.5 \times 10^{-6}$ & $3.4 \times 10^{-15}$ & $1.6 \times 10^{-10}$ & $2.2 \times 10^{-5}$ \\
\hline 4 & 0 & $4.3 \times 10^{-17}$ & $1.2 \times 10^{-11}$ & $3.5 \times 10^{-6}$ & $4.2 \times 10^{-16}$ & $2.2 \times 10^{-11}$ & $1.9 \times 10^{-5}$ \\
\hline 4 & 1 & $1.2 \times 10^{-16}$ & $6.4 \times 10^{-11}$ & $1.9 \times 10^{-6}$ & $1.1 \times 10^{-15}$ & $4.0 \times 10^{-11}$ & $2.9 \times 10^{-5}$ \\
\hline 4 & 2 & $1.8 \times 10^{-16}$ & $6.6 \times 10^{-11}$ & $2.7 \times 10^{-6}$ & $1.6 \times 10^{-15}$ & $7.9 \times 10^{-11}$ & $2.0 \times 10^{-5}$ \\
\hline 4 & 3 & $2.1 \times 10^{-16}$ & $1.7 \times 10^{-10}$ & $1.2 \times 10^{-6}$ & $1.8 \times 10^{-15}$ & $1.8 \times 10^{-10}$ & $9.7 \times 10^{-6}$ \\
\hline & $P_{10}$ & & & $7.2 \times 10^{-6}$ & & & $3.0 \times 10^{-5}$ \\
\hline \multicolumn{8}{|c|}{$T=300 \mathrm{~K}$} \\
\hline 1 & 0 & $2.9 \times 10^{-15}$ & $1.3 \times 10^{-10}$ & $2.3 \times 10^{-5}$ & $3.2 \times 10^{-15}$ & $1.1 \times 10^{-10}$ & $3.0 \times 10^{-5}$ \\
\hline 2 & 0 & $1.2 \times 10^{-15}$ & $3.3 \times 10^{-11}$ & $3.7 \times 10^{-5}$ & $2.0 \times 10^{-15}$ & $2.6 \times 10^{-11}$ & $7.7 \times 10^{-5}$ \\
\hline 2 & 1 & $3.1 \times 10^{-15}$ & $1.5 \times 10^{-10}$ & $2.0 \times 10^{-5}$ & $5.8 \times 10^{-15}$ & $1.6 \times 10^{-10}$ & $3.7 \times 10^{-5}$ \\
\hline 3 & 0 & $4.9 \times 10^{-16}$ & $2.8 \times 10^{-11}$ & $1.7 \times 10^{-5}$ & $1.3 \times 10^{-15}$ & $2.5 \times 10^{-11}$ & $5.2 \times 10^{-5}$ \\
\hline 3 & 1 & $1.3 \times 10^{-15}$ & $7.2 \times 10^{-11}$ & $1.9 \times 10^{-5}$ & $3.5 \times 10^{-15}$ & $6.1 \times 10^{-11}$ & $5.8 \times 10^{-5}$ \\
\hline 3 & 2 & $1.7 \times 10^{-15}$ & $2.2 \times 10^{-10}$ & $7.8 \times 10^{-6}$ & $5.1 \times 10^{-15}$ & $1.6 \times 10^{-10}$ & $3.2 \times 10^{-5}$ \\
\hline 4 & 0 & $2.1 \times 10^{-16}$ & $1.2 \times 10^{-11}$ & $1.7 \times 10^{-5}$ & $7.5 \times 10^{-16}$ & $1.8 \times 10^{-11}$ & $4.2 \times 10^{-5}$ \\
\hline 4 & 1 & $5.3 \times 10^{-16}$ & $6.9 \times 10^{-11}$ & $7.6 \times 10^{-6}$ & $2.1 \times 10^{-15}$ & $4.6 \times 10^{-11}$ & $4.6 \times 10^{-5}$ \\
\hline 4 & 2 & $7.6 \times 10^{-16}$ & $6.9 \times 10^{-11}$ & $1.1 \times 10^{-5}$ & $3.0 \times 10^{-15}$ & $6.9 \times 10^{-11}$ & $4.4 \times 10^{-5}$ \\
\hline 4 & 3 & $8.1 \times 10^{-16}$ & $1.8 \times 10^{-10}$ & $4.5 \times 10^{-6}$ & $3.5 \times 10^{-15}$ & $1.8 \times 10^{-10}$ & $2.0 \times 10^{-5}$ \\
\hline \multicolumn{4}{|c|}{ Average $P_{10}$} & $1.6 \times 10^{-5}$ & & & $4.4 \times 10^{-05}$ \\
\hline \multicolumn{8}{|c|}{$T=400 \mathrm{~K}$} \\
\hline 1 & 0 & $3.2 \times 10^{-15}$ & $1.3 \times 10^{-10}$ & $2.6 \times 10^{-5}$ & $3.3 \times 10^{-15}$ & $1.1 \times 10^{-10}$ & $3.1 \times 10^{-5}$ \\
\hline 2 & 0 & $1.6 \times 10^{-15}$ & $3.4 \times 10^{-11}$ & $4.8 \times 10^{-5}$ & $2.2 \times 10^{-15}$ & $2.5 \times 10^{-11}$ & $8.8 \times 10^{-5}$ \\
\hline 2 & 1 & $4.0 \times 10^{-15}$ & $1.6 \times 10^{-10}$ & $2.5 \times 10^{-5}$ & $6.5 \times 10^{-15}$ & $1.6 \times 10^{-10}$ & $4.0 \times 10^{-5}$ \\
\hline 3 & 0 & $7.2 \times 10^{-16}$ & $3.0 \times 10^{-11}$ & $2.4 \times 10^{-5}$ & $1.6 \times 10^{-15}$ & $2.6 \times 10^{-11}$ & $5.9 \times 10^{-5}$ \\
\hline 3 & 1 & $2.0 \times 10^{-15}$ & $7.1 \times 10^{-11}$ & $2.8 \times 10^{-5}$ & $4.2 \times 10^{-15}$ & $5.7 \times 10^{-11}$ & $7.3 \times 10^{-5}$ \\
\hline 3 & 2 & $2.5 \times 10^{-15}$ & $2.2 \times 10^{-10}$ & $1.1 \times 10^{-5}$ & $6.2 \times 10^{-15}$ & $1.6 \times 10^{-10}$ & $3.7 \times 10^{-5}$ \\
\hline 4 & 0 & $3.3 \times 10^{-16}$ & $1.2 \times 10^{-11}$ & $2.7 \times 10^{-5}$ & $9.2 \times 10^{-16}$ & $1.6 \times 10^{-11}$ & $5.6 \times 10^{-5}$ \\
\hline 4 & 1 & $8.5 \times 10^{-16}$ & $7.1 \times 10^{-11}$ & $1.2 \times 10^{-5}$ & $2.7 \times 10^{-15}$ & $4.9 \times 10^{-11}$ & $5.4 \times 10^{-5}$ \\
\hline 4 & 2 & $1.2 \times 10^{-15}$ & $7.1 \times 10^{-11}$ & $1.8 \times 10^{-5}$ & $3.9 \times 10^{-15}$ & $6.8 \times 10^{-11}$ & $5.7 \times 10^{-5}$ \\
\hline 4 & 3 & $1.3 \times 10^{-15}$ & $1.9 \times 10^{-10}$ & $7.1 \times 10^{-6}$ & $4.6 \times 10^{-15}$ & $1.8 \times 10^{-10}$ & $2.5 \times 10^{-5}$ \\
\hline \multicolumn{4}{|c|}{ Average $P_{10}$} & $2.3 \times 10^{-5}$ & & & $5.2 \times 10^{-5}$ \\
\hline
\end{tabular}

Notes. The $P_{10}$ factors (see Eq. (7)) are also reported. Tables with the rates for the rotational transitions for both isotopes $\left({ }^{36} \mathrm{ArH}^{+}\right.$and $\left.{ }^{36} \mathrm{ArD}^{+}\right)$by collision with $\mathrm{He}$ are available at the CDS.

two vibrational states. Typical de-excitation cross sections are shown in Fig. 1C, while Fig. 2A shows the $|\Delta j|=1$ rotational rate coefficients in the vibrational state $v=0$ at $10 \mathrm{~K}$. The agreement between both sets of data is excellent for the cross sections and therefore for the rate coefficients, confirming the validity of the rigid rotor approach for determining the rotational rate coefficients. The rates computed in this work are also compared with the rates of Bop et al. (2017) (see Fig. 2). Only small differences at low values of the initial rotational states $\left(j_{i} \leq 6\right)$ were found. However, significant discrepancies are observed from the initial rotational numbers $j_{\mathrm{i}}>6$, Fig. 2A. Even if such disagreements reduce with the increment of the temperature, at $T=10 \mathrm{~K}$, they can be of one order of magnitude. Further analyses of the rates for transitions from the initial rotational state $j=4$ and $j=5$ are shown in Fig. 2B and C. In these cases, the difference between the rates computed in this work and those of Bop et al. (2017) are significant from $|\Delta j|>2$; see for example the $|\Delta j|=4$ transition in panel $\mathrm{C}$, where the ratio between both sets of data is 4.3 . However, the differences in the rates increase as the rates decreases. It is worth pointing out again that the global minimum of our PES is less deep than that of Bop et al. (2017). However, such a difference (around $2 \%$ ), does not explain the observed disagreements. Our calculations also show that the use of the rigid rotor approximation is not a possible cause of the differences. Even if a comparison with the $V_{\lambda}(R)$ coefficients of the expansion of both surfaces, $V\left(R, \theta, r=r_{\mathrm{e}}\right)=\sum_{\lambda} V_{\lambda}(R) P_{\lambda}(\cos \theta)$, does not show differences between them; Fig. 4 in Bop et al. (2017) only shows those up to $\lambda=3$ and the differences in the anisotropy of the PESs could be related with those $V_{\lambda}(R)$ for $\lambda \geq 4$. Furthermore, 
if the rate coefficients computed in this work are compared with those with the most common colliders in the ISM, it can be seen that they are at the same order of magnitude as those computed for the collision with $H$ (Dagdigian 2018), while the rates for the collision of $\mathrm{ArH}^{+}$with $e^{-}$(Hamilton et al. 2016) are a factor around $10^{3}$ higher than those for $\mathrm{ArH}^{+}+\mathrm{He}$. For the case of collisions with $\mathrm{H}_{2}$, there are not rotational rates reported so far, however, for $\mathrm{ArH}^{+}+\mathrm{H}_{2}$, reactive processes should also be considered (Villinger et al. 1982).

The electronic structure of the different isotopes of the $\mathrm{ArH}^{+}+\mathrm{He}$ system is the same. The RKHS PES can be used with a small modification in the Jacobi coordinates to take into account the change in the center of mass of the diatom. Figure 3 shows the rotational rate coefficients for the isotopes ${ }^{36} \mathrm{ArH}^{+}$, ${ }^{38} \mathrm{ArH}^{+}$, and ${ }^{40} \mathrm{ArH}^{+}$in collision with He. The effects on the rates for different isotopologs of $\mathrm{Ar}$ are negligible, and the values computed for one isotope can be used for the others. The calculations of the rates for ${ }^{36} \mathrm{ArD}^{+}+\mathrm{He}$ showed different behavior. The rotational levels of the ${ }^{36} \mathrm{ArD}^{+}$(its rotational constant, $B_{\mathrm{e}}$, is $5.295 \mathrm{~cm}^{-1}$ ) differ significantly from those of its isotopolog ${ }^{36} \mathrm{ArH}^{+}\left(B_{\mathrm{e}}=10.273 \mathrm{~cm}^{-1}\right)$, as well as the reduced mass of the systems. Hence, the significant differences found between the rates of ${ }^{36} \mathrm{ArH}^{+}$and ${ }^{36} \mathrm{ArD}^{+}$are not a surprise. In rotational transitions, such as $j=2 \rightarrow j^{\prime}=1$, the rates for the deuterated species are higher than those for the hydrogenated species, for other transitions (e.g., $j=3 \rightarrow j^{\prime}=2$ ) the rates of ${ }^{36} \mathrm{ArD}^{+}+\mathrm{He}$ are lower than those of ${ }^{36} \mathrm{ArH}^{+}+\mathrm{He}$, while in several cases, the rates depend on the temperature (e.g., $j=1 \rightarrow j^{\prime}=0$ ). Therefore, one set of rates cannot be straightforwardly estimated from the other. The rotational rates up to $j=12$ in the ground vibrational states from 10 to $500 \mathrm{~K}$ (which are available at the CDS) would be valuable in the interpretation of the physical-chemical conditions of the regions of the ISM, where $\mathrm{ArH}^{+}$are detected and also in the analysis of the regions of possible future detection of $\mathrm{ArD}^{+}$.

An extra set of close coupling calculations from the initial vibrational state $v=1$ for the lower rotational states was also performed. Table 1 shows the rates for the rotational transitions in $v=0$ state of ${ }^{36} \mathrm{ArH}$ and ${ }^{36} \mathrm{ArD}$, and those obtained for the same rotational transitions between the vibrational levels $v=1$ and $v=0$ at several temperatures. Furthermore, the proportionality factor $P_{v, v^{\prime}}$ of the rates in different vibrational levels and those in the ground vibrational states (Chandra \& Sharma 2001),

$k\left(T \mid v, j \rightarrow v^{\prime}, j^{\prime}\right)=P_{v, v^{\prime}} k\left(T \mid 0, j \rightarrow 0, j^{\prime}\right)$,

was evaluated. Since $v=1$ and $v^{\prime}=0$, the $P_{1,0}$ factor was included in this table. It is worth noting that $P_{1,0}$ varies slowly with $T$. The use of the average value improves with the increment of $T$. These values, together with the rotational rates, will be useful if any of the $\mathrm{ArH}^{+}$isotopologs are detected in an excited vibrational state to analyze the physical conditions of the region where this would be observed (Chandra \& Sharma 2001). The collisional set of rate coefficients for $\mathrm{ArH}^{+}+\mathrm{He}$ were extended up to $v=1$ and $j=12$ and can be found at the CDS.

\section{Conclusions}

The first three-dimensional PES for the $\mathrm{ArH}^{+}+\mathrm{He}$ system was developed in this work. Two sets of $a b$ initio energies at the CCSD(T)/aug-cc-pVQZ and CCSD(T)/aug-cc-pV5Z level were computed and the energies at the CBS limit were determined. The analytical surface was represented using a kernel-based method. Two minima at the linear configurations $\mathrm{He}-\mathrm{ArH}^{+}$and $\mathrm{ArH}^{+}-\mathrm{He}$ were found and compared with a previous two-dimensional PES. This new surface was used in the determination of the lower rotational rate coefficients for the collision of $\mathrm{ArH}^{+}$with $\mathrm{He}$, which showed significant differences with previous data. The isotopic effects were evaluated from close-coupling calculations. Differences were found only for the deuterated species, while for the different isotopologs of Ar, the effects are negligible. The rates for the rovibrational transitions were also analyzed. Finally, new sets of rotational rates coefficients for ${ }^{36} \mathrm{ArH}^{+}+\mathrm{He}$ and ${ }^{36} \mathrm{ArD}^{+}+\mathrm{He}$ were reported.

Acknowledgements. Support from the projects CONICYT/FONDECYT/ INICIACION/No. 11160005 and Programa Ciencias Básicas Proyecto No. p223LH001 -108 are acknowledged. O. D-A. gratefully acknowledge Cheikh T. Bop for sending us the previous rates for the $\mathrm{ArH}^{+}+\mathrm{He}$ system by email.

\section{References}

Abdoulanziz, A., Colboc, F., Little, D. A., et al. 2018, MNRAS, 479, 2415 Anicich, V. G. 1993, J. Phys. Chem. Ref. Data, 22, 1469

Arthurs, A. M., \& Dalgarno, A. 1960, Proc. R. Soc. London Ser. A, 256, 540

Barlow, M., Swinyard, B., Owen, P., et al. 2013, Science, 342, 1343

Bop, C. T., Hammami, K., Niane, A., Faye, N., \& Jaïdane, N. 2017, MNRAS, 465, 1137

Boys, S. F., \& Bernardi, F. 1970, Mol. Phys., 19, 553

Buffa, G. 2012, MNRAS, 421, 719

Buffa, G., Dore, L., \& Meuwly, M. 2009, MNRAS, 397, 1909

Burcl, R., Chałasiński, G., Bukowski, R., \& Szczȩśniak, M. M. 1995, J. Chem. Phys., 103, 1498

Chandra, S., \& Sharma, A. 2001, A\&A, 376, 356

Colbert, D. T., \& Miller, W. H. 1992, J. Chem. Phys., 96, 1982

Dagdigian, P. J. 2018, MNRAS, 477, 802

Denis-Alpizar, O., Stoecklin, T., \& Halvick, P. 2015, MNRAS, 453, 1317

Denis-Alpizar, O., Bemish, R. J., \& Meuwly, M. 2017, Phys. Chem. Chem. Phys., 19, 2392

Denis-Alpizar, O., Trabelsi, T., Hochlaf, M., \& Stoecklin, T. 2018, MNRAS, 475,783

Dumouchel, F., Kłos, J., Toboła, R., et al. 2012, J. Chem. Phys., 137, 114306

Dunning, T. H. 1989, J. Chem. Phys., 90, 1007

Halkier, A., Klopper, W., Helgaker, T., Jorgensen, P., \& Taylor, P. R. 1999, J. Chem. Phys., 111, 9157

Hamilton, J. R., Faure, A., \& Tennyson, J. 2016, MNRAS, 455, 3281

Ho, T. S., \& Rabitz, H. 1996, J. Phys. Chem., 104, 2584

Linstrom, P. J., \& Mallard, W. G. 2001, J. Chem. Eng. Data, 46, 1059

Loh, E. D., Baldwin, J. A., Ferland, G. J., et al. 2012, MNRAS, 421, 789

Manolopoulos, D. E. 1988, PhD Thesis, University of Cambridge, UK

Mertens, L. A., Labiad, H., Denis-Alpizar, O., et al. 2017, Chem. Phys. Lett., 683,521

Müller, H. S., Muller, S., Schilke, P., et al. 2015, A\&A, 582, L4

Novak, C. M., \& Fortenberry, R. C. 2016, J. Mol. Spectrosc., 322, 29

Priestley, F., Barlow, M., \& Viti, S. 2017, MNRAS, 472, 4444

Schilke, P., Neufeld, D. A., Müller, H. S., et al. 2014, A\&A, 566, A29

Schöier, F. L., van der Tak, F. F. S., van Dishoeck, E. F., \& Black, J. H. 2005 , A\&A, 432, 369

Soldán, P., \& Hutson, J. M. 2000, J. Chem. Phys., 112, 4415

Stoecklin, T., Voronin, A., \& Rayez, J. C. 2002, Phys. Rev. A, 66, 042703

Villinger, H., Futrell, J., Howorka, F., Duric, N., \& Lindinger, W. 1982, J. Chem. Phys., 76, 3529

Werfelli, G., Balança, C., Stoecklin, T., Kerkeni, B., \& Feautrier, N. 2017, MNRAS, 468, 2582

Werner, H.-J., Knowles, P. J., Knizia, G., Manby, F. R., \& Schütz, M. 2012, WIREs Comput. Mol. Sci., 2, 242

Wyatt, J., Strattan, L., Snyder, S., \& Hierl, P. M. 1975, J. Chem. Phys., 62, 2555 